\title{
THE RELEVANCE TO ASTROPHYSICS OF \\ THE RESULTS OF RECENT EXPERIMENTS WITH \\ COLLIDING CHARGED-PARTICLE BEAMS
}

\author{
K. T. DOLDER
}

Dept. of Atomic Physics, University of Newcastle upon Tyne, England

\begin{abstract}
A summary is given of some recent measurements of the ionization and excitation of positive ions by electrons, and the detachment of electrons from $\mathrm{H}^{-}$by proton and electron impacts. The results are discussed and compared with theory. Conclusions are drawn which are relevant to atomic collision processes which occur in astrophyscial plasmas.
\end{abstract}

\section{Introduction}

In the tenuous outer layers of stars the mean free path of radiation usually exceeds the thickness of the atmosphere. The laws of equilibrium thermodynamics cannot therefore be applied and one must have knowledge of the various atomic processes which occur within the atmosphere before its properties can be fully understood. For example, a calculation of the electron temperature of the solar corona requires cross sections for the ionization and recombination of predominant ions as functions of the energy of incident electrons.

During the last decade crossed beam experiments have been performed to study collisions between charged particles by experimental methods which have been reviewed by Harrison (1968), Dunn (1969) and Dolder (1969). These experiments have, so far, only been performed with singly- or double-charged ions so that the results are not usually directly applicable to stellar atmospheres. The present paper will draw attention to results which may nevertheless interest astrophysicists. It will discuss measurements of the ionization and excitation of ions by electron impact and the detachment of electrons from $\mathrm{H}^{-}$by collisions with protons or electrons.

\section{The Ionization of Positive Ions by Electron Impact}

Consider the process,

$$
\mathrm{A}^{p+}+e \rightarrow \mathrm{A}^{(p+1)+}+2 e .
$$

In the absence of a detailed calculation, the cross-section $(\sigma)$ can be estimated from Thomson's classical result,

$$
\sigma=\frac{n \pi e^{4}}{E^{2}}\left(\frac{E}{\chi}-1\right)
$$

where $\chi$ is the ionization energy of $\mathrm{A}^{p+}, E$ is the energy of the incident electron, $n$ is the number of electrons in the shell from which ionization occurs, and $e$ represents 
the electronic charge. In spite of the crude assumptions on which Equation (2) is based, it often gives results which agree within a factor two or three with experiment, although it is sometimes spectacularly misleading, as in the case of electron detachment from $\mathrm{H}^{-}$. It follows from (2) that cross-sections of two isoelectronic ions are simply related by,

$$
\frac{\sigma_{1}}{\sigma_{2}}=\left(\frac{\chi_{2}}{\chi_{1}}\right)^{2}
$$

provided that the incident electron energies are expressed in terms of the respective ionization energies. This simple scaling law is widely used and it is contained in some of the empirical formulae (e.g. Drawin, 1961) which are sometimes employed to estimate cross-sections of highly-charged ions which are inaccessible to measurement. It has recently been possible to test the validity of classical scaling, and Figure 1 shows four examples of the measured cross-section of an ion compared with classicallyscaled measurements of its isoelectronic atom. It can be seen that each of the pairs, $\mathrm{H}, \mathrm{He}^{+} ; \mathrm{He}, \mathrm{Li}^{+}$and $\mathrm{A}, \mathrm{K}^{+}$obey the scaling law, although in each case the positive ion has a slightly larger cross-section for slower electrons (presumably a consequence of its coulomb attraction). Figure 1d, however, illustrates that classical scaling is certainly not universally valid, although in the neon sequence $\left(\mathrm{Ne}, \mathrm{Na}^{+}, \mathrm{Mg}^{2+}\right)$ it improves quite rapidly with increasing nuclear charge.

Quantum calculations of the ionization cross-sections of complex atoms frequently
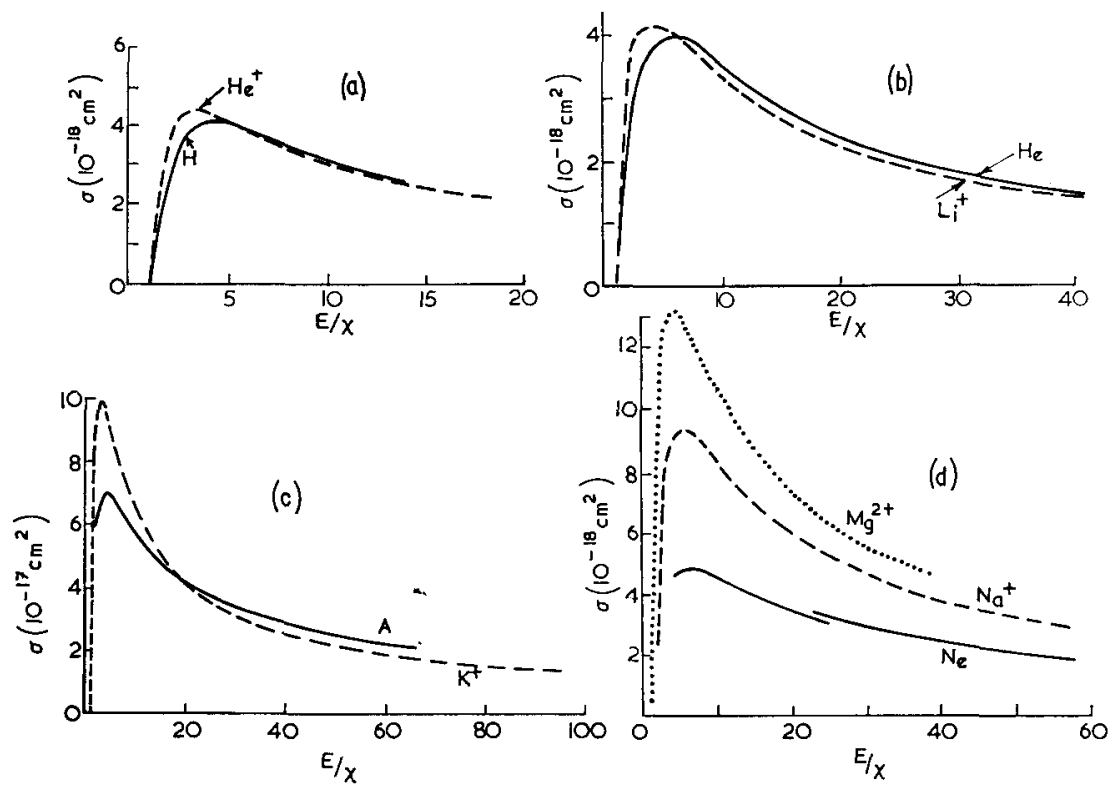

Fig. 1. Measured cross-sections for the ionization of $\mathrm{He}^{+}, \mathrm{Li}^{+}, \mathrm{K}^{+}$and $\mathrm{Mg}^{2+}$ ions compared with classically scaled measurements of their isoelectronic atoms. The energies of incident electrons are expressed in terms of the respective ionization energies. 
differ substantially from experimental results (e.g., Peach, 1968, 1970). This is illustrated by Figure 2a which compares calculated (Peach, 1968) and measured (Schram et al., 1966a, b) cross-sections for neon. Even at energies for which Born's approximation should be valid, there are discrepancies amounting almost to a factor of two. The corresponding differences are however much smaller for the more highly-charged members of the neon sequence. This can be seen from Figures $2 b$ and $2 c$ which compare the Coulomb-Born calculations of Moores and Nussbaumer (private communication, 1970a) with measurements for $\mathrm{Na}^{+}$(Peart and Dolder, 1968a) and $\mathrm{Mg}^{2+}$ (Peart et al., 1969a). It seems that the wavefunctions used take insufficient account of electron correlation (interaction between electrons in the same subshell) and that these effects become less important, relative to the nuclear field, as the nuclear charge increases.

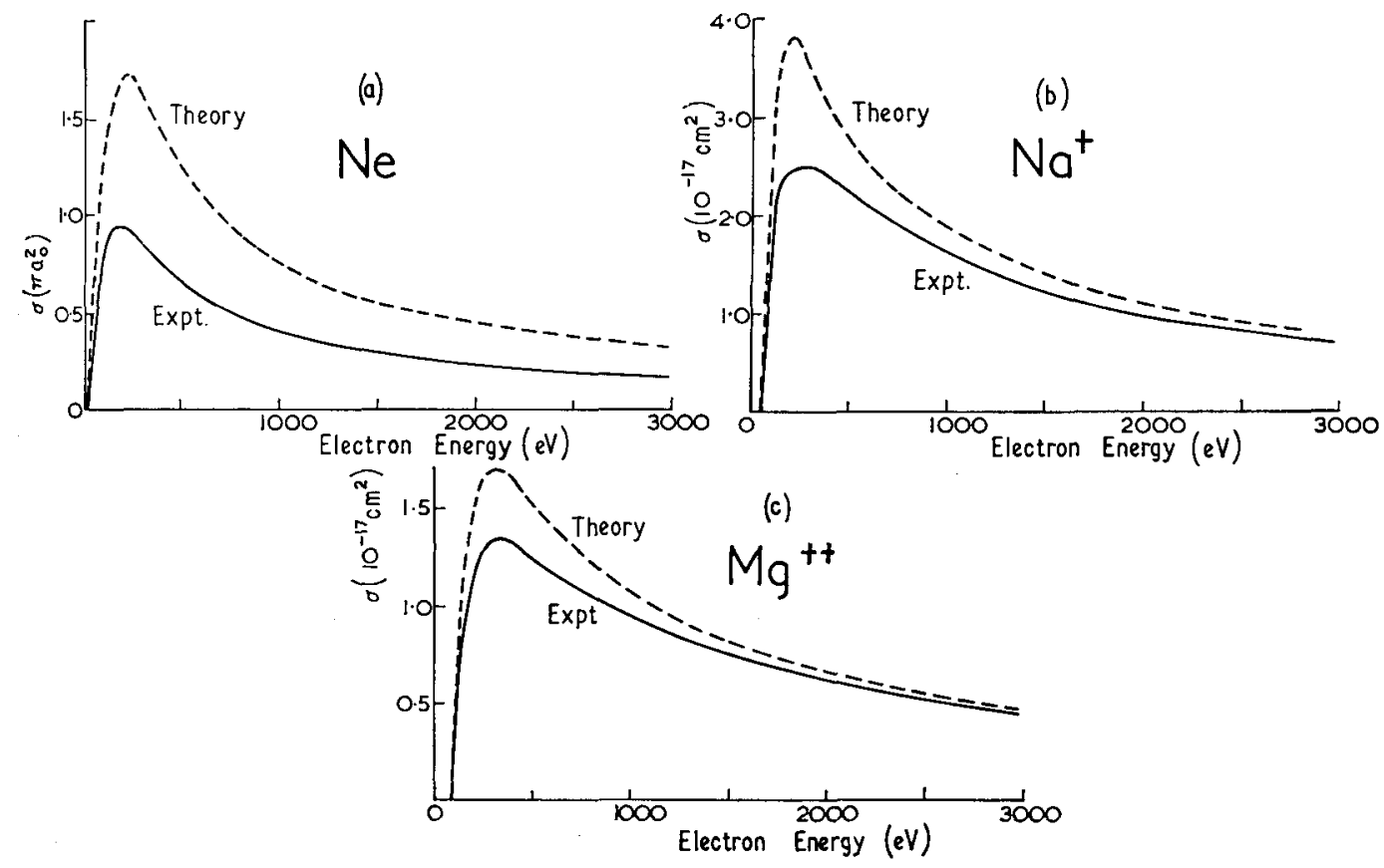

Fig. 2. Comparison of measured and calculated electron impact cross-sections for the first three members of the neon isoelectronic sequence.

The results in Figures 1 and 2 suggest that the accuracy of theory and the classical scaling law should both improve very significantly as one proceeds to the more highlycharged ions which are of astrophysical interest. Unfortunately, these simplifications may sometimes be offset by contributions to the ionization cross-section from inner shell electrons and one must consider both autoionization and direct inner shell ionization. Autoionization occurs if an inner shell electron is excited and then decays, without the emission of radiation, so that its energy is used to eject a more loosely- 
bound electron. Bely (1968) discussed the autoionization of sodium-like ions and suggested that it may have appreciable astrophysical consequences especially since the contribution should increase with nuclear charge. Although it is clear that Bely overestimated the magnitude of this contribution, at least for sodium (e.g. McFarland and Kinney, 1964) and $\mathrm{Mg}^{+}$(Martin et al., 1968), it cannot be assumed that these effects are negligible for more highly-charged sodium-like ions. A calculation in the Coulomb-Born approximation by Moores and Nussbaumer (1970b) shows appreciable autoionization even for $\mathrm{Mg}^{+}$(larger, in fact, than can be reconciled with experiment, see Figure 3). This process clearly deserves further attention, although theory encounters the problem that autoionization tends to be largest at near-threshold energies where Born's approximation is unreliable.

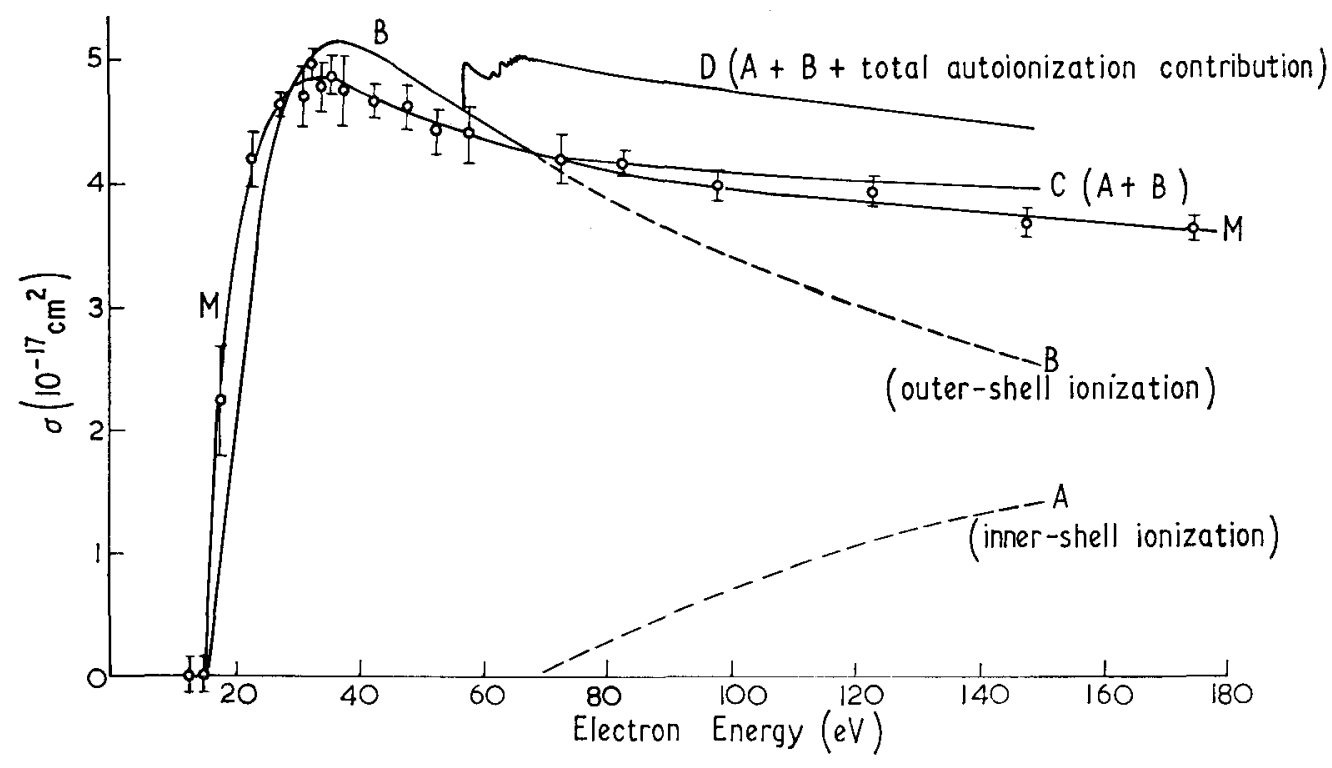

Fig. 3. Calculated and measured $(M)$ cross-sections for the ionization of $\mathrm{Mg}^{+}$ions by electrons. Curve $C$ represents the sum of calculated direct ionization from inner $(A)$ and outer $(B)$ shells. Curve $D$ also includes the calculated autoionization contribution.

Moores and Nussbaumer (1970b) have also established that there is considerable direct inner-shell ionization of $\mathrm{Mg}^{+}$. There is excellent agreement (Figure 3) between their calculation and experiment only when inner shell ionization is included.

The Born and Bethe approximations not only provide the basis for most of the theory of ionization but the proportionality (implied by Bethe's approximation),

$$
\sigma E \propto \log E+\text { constant }
$$

is often included in empirical expressions for ionization cross-sections. Both approximations are expected to be valid only for fast electrons and the ranges of validity can be established by comparing experiment with theory. Figures 4 and 5 show 

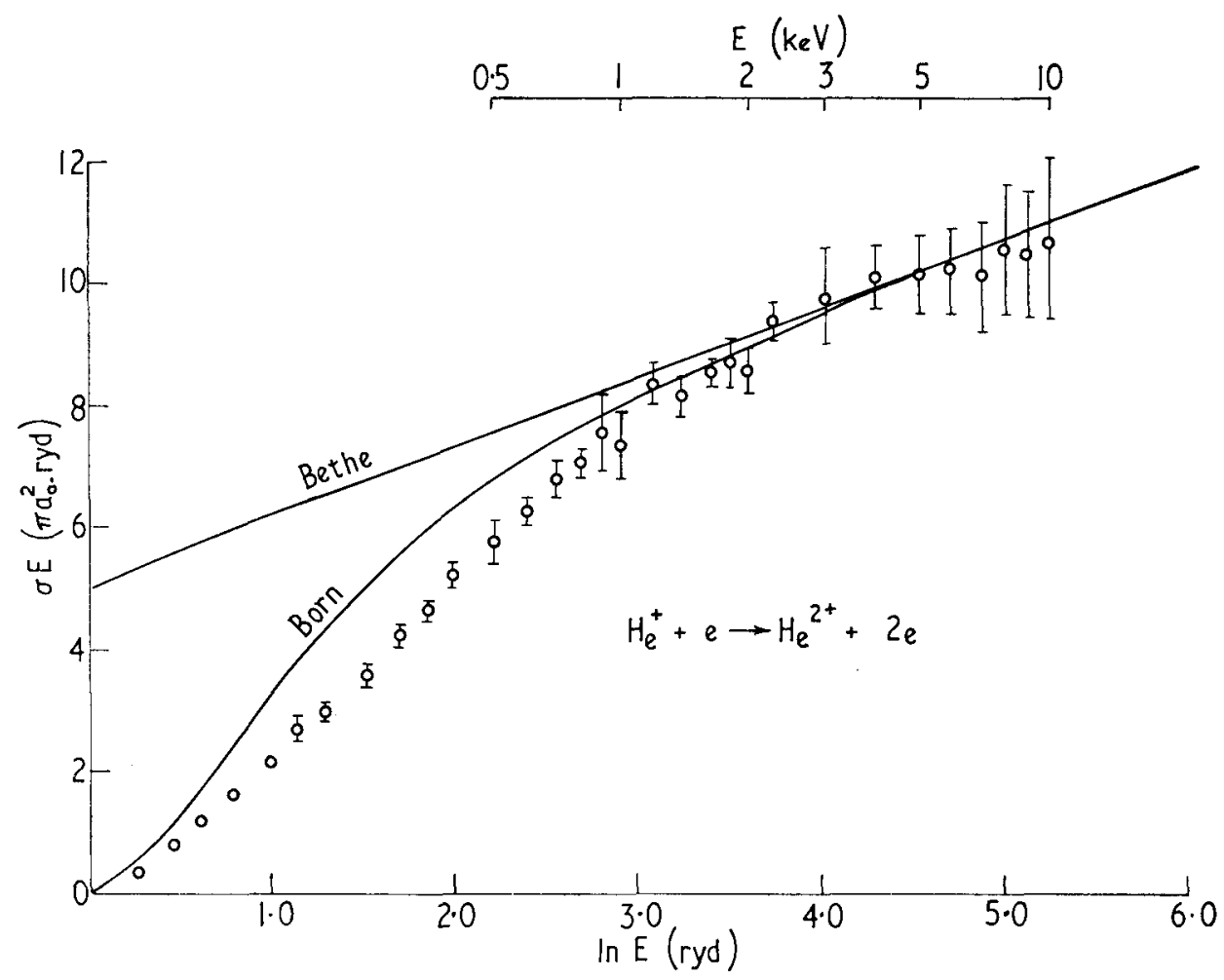

Fig. 4. Measured cross-sections $(\sigma)$ for the ionization of $\mathrm{He}^{+}$ions by electrons compared with predictions of the Born and Bethe approximations.

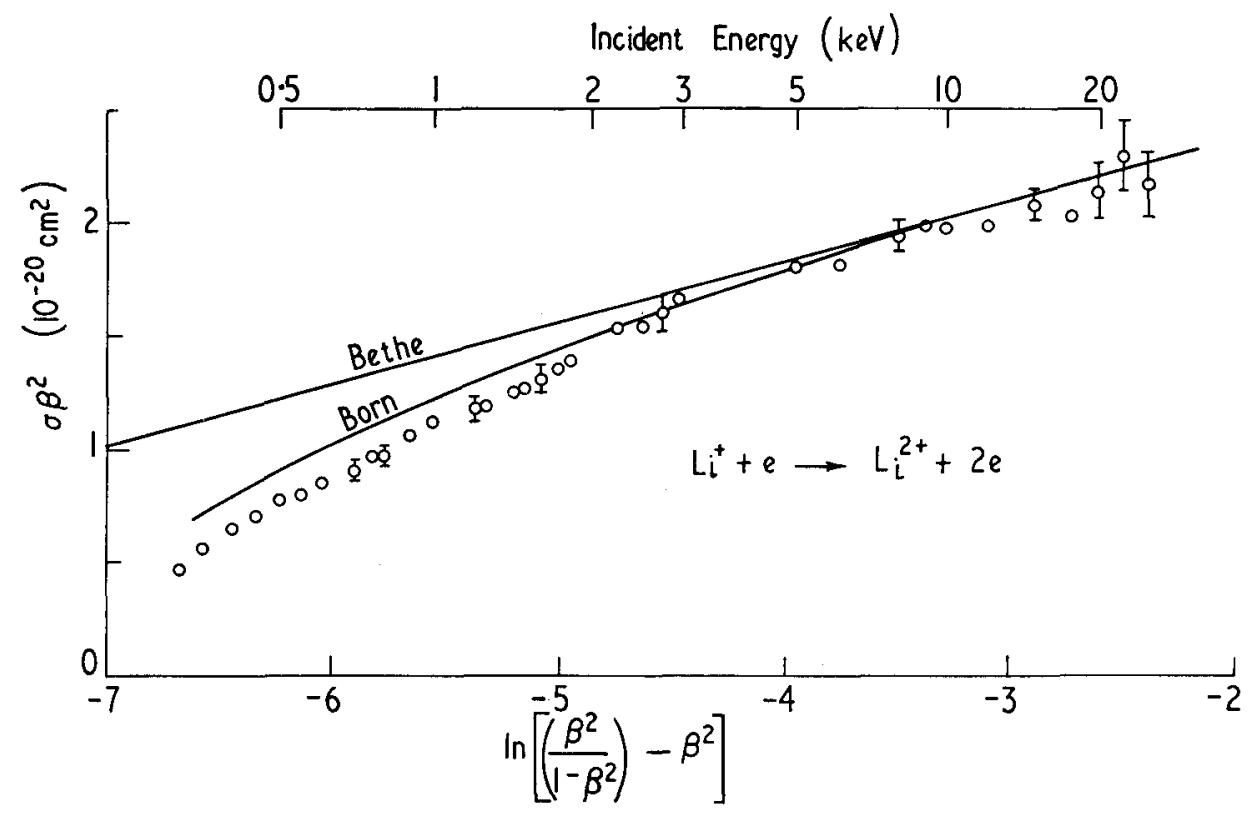

Fig. 5. Measured cross-sections $(\sigma)$ for the ionization of $\mathrm{Li}^{+}$ions compared with predictions of the Born and Bethe approximations. 
measurements (Peart et al., 1969b) for the single ionization of $\mathrm{He}^{+}$and $\mathrm{Li}^{+}$and the results of corresponding theories in the Born and Bethe approximations. The calculation for $\mathrm{He}^{+}$were performed by Omidvar (1969) whilst those for $\mathrm{Li}^{+}$were due to Kim and Inokuti (Bethe approximation, 1969), and Moores and Nussbaumer (Coulomb-Born approximation, 1970b; see also Economides and McDowell, 1969). In both cases Born's approximation is valid for incident electron energies greater than twenty times threshold whilst Bethe's approximation holds only at more than fifty times threshold; it is not however suggested that these criteria are necessarily valid for other ions. Such high electron energies $(\$ 25 \mathrm{keV})$ were used in the experiments with $\mathrm{Li}^{+}$that relativistic effects were appreciable. The results in Figure 6 have therefore been plotted to give a linear relation in the relativistic Bethe approximation and the symbol $\beta$ represents the electron velocity relative to that of light.

A list of experiments on the ionization of positive ions is included in the review by Dolder. To this list can be added the more recent experiments by Aitken and Harrison (private communication) on the single ionization of $\mathrm{C}^{+}, \mathrm{N}^{2+}, \mathrm{O}^{+}$and $\mathrm{O}^{2+}$.

\section{The Excitation of Positive Ions by Electron Impact}

Absolute cross-sections have been measured by Bacon and Hooper (1969) for,

$$
\begin{aligned}
& \mathrm{Ba}^{+}\left(6^{2} S_{1 / 2}\right)+e \rightarrow \mathrm{Ba}^{+}\left(6^{2} P_{1 / 2}^{0}\right)+e(\lambda=4934 \AA) \\
& \mathrm{Ba}^{+}\left(6^{2} S_{1 / 2}\right)+e \rightarrow \mathrm{Ba}^{+}\left(6^{2} P_{3 / 2}^{0}\right)+e(\lambda=4554 \AA)
\end{aligned}
$$

and by Lee and Carleton (private communication, 1969) for,

$$
\mathrm{N}_{2}^{+}+e \rightarrow \mathrm{N}_{2}^{+}+e+h v \quad(\lambda=3914 \AA) .
$$

The measured cross sections of the former reaction are compared with semi-empirical theory in Figure 6. In each experiment the prime experimental difficulty was the absolute measurement of the flux of photons produced. Absolute cross sections can also be deduced by normalising measurements to the results of Born's approximation at high energies, if it is assumed that theory converges to the first Born approximation. This procedure is likely to be accurate only for simple ions, such as $\mathrm{He}^{+}$, which have accurate wavefunctions and Dance et al. (1966) used this normalisation to obtain absolute cross sections for,

$$
\mathrm{He}^{+}(1 S)+e \rightarrow \mathrm{He}^{+}(2 S)+e \quad(\lambda=304 \AA) .
$$

A special case of excitation was the autoionization of $\mathrm{Ba}^{+}$observed by Peart and Dolder (1968b). Their results, and those of Dance et al., illustrate the abrupt rise of the excitation function from threshold which is characteristic of positive ions. Figure 7 presents four measurements of the ionization function of $\mathrm{Ba}^{+}$near the autoionization threshold $(\approx 17 \mathrm{eV})$. The sudden rise between 16 and $18 \mathrm{eV}$ is attributed to the onset of autoionization and the steepness of the measured autoionization function appears to be limited only by the spread of energies $( \pm 2 \mathrm{eV})$ of the electrons used in the experiment. A measurement of this spread is illustrated by the inset. 


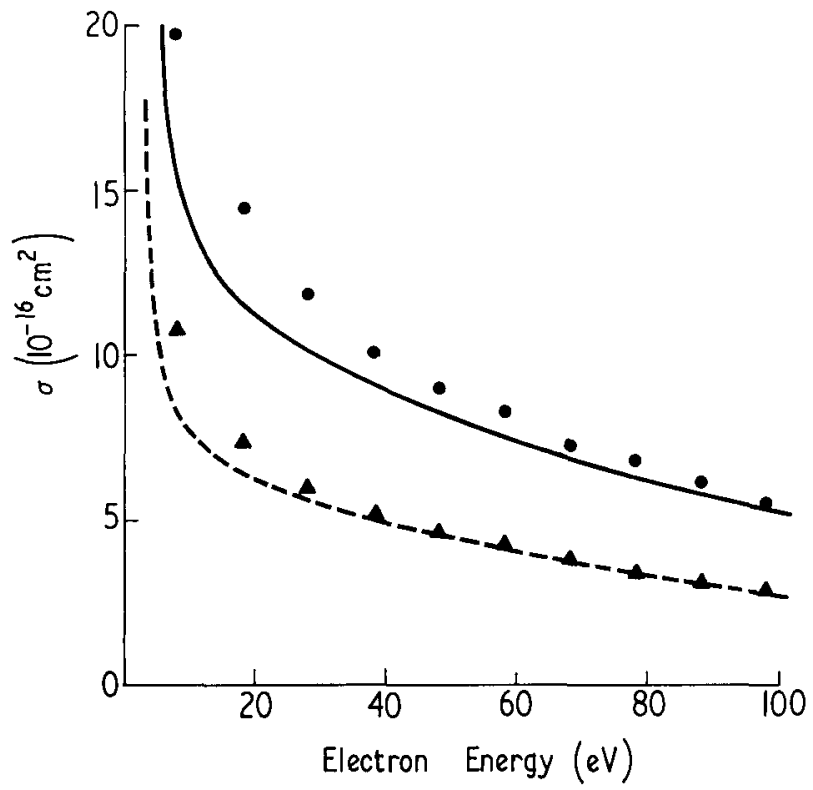

Fig. 6. Measured absolute cross-sections for the excitation of $\mathrm{Ba}^{+}$ions by electrons compared with the results of semi-empirical theory. The circles and the continuous curve refer to the transition $\left(6^{2} S_{1 / 2}-6^{2} P^{0} 0_{3 / 2}\right)$ whilst the other results refer to $\left(6^{2} S_{1 / 2}-6^{2} P^{0} 1 / 2\right)$.
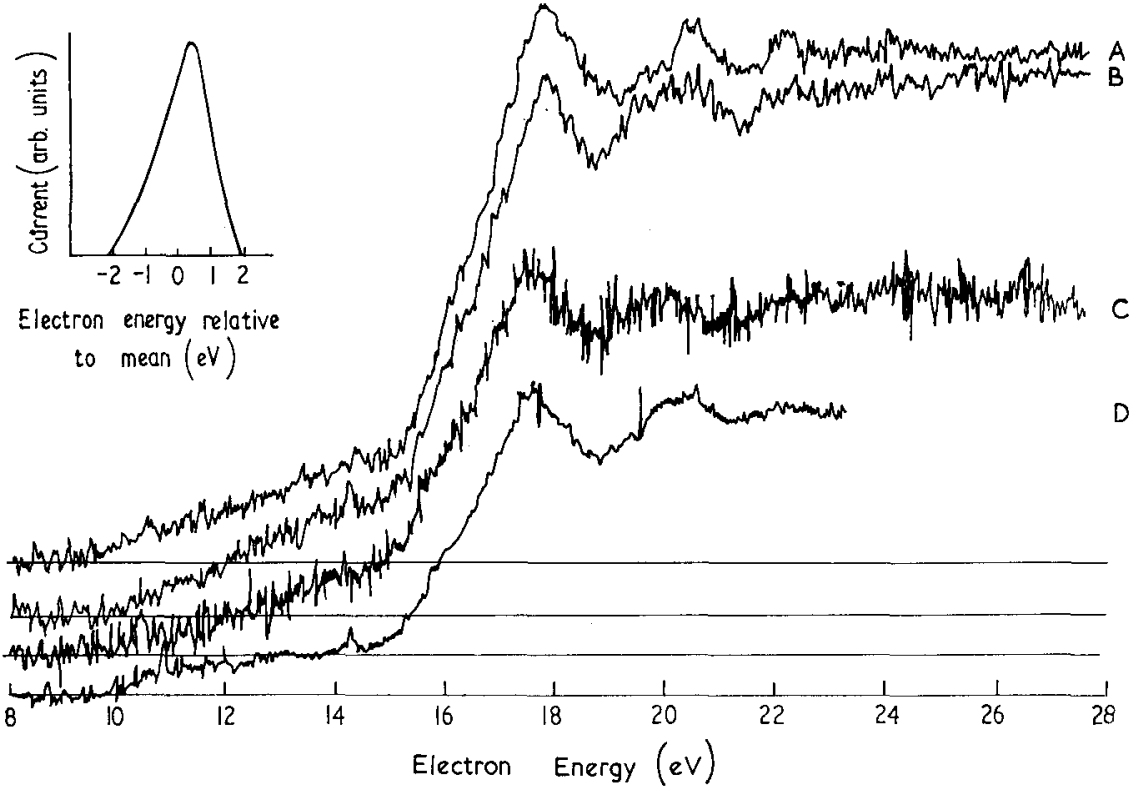

Fig. 7. Four measurements of the ionization function of $\mathrm{Ba}^{+}$ions by electrons. Each trace shows an abrupt rise for electron energies close to $16 \mathrm{eV}$. This is attributed to the onset of autoionization. There is also evidence of structure above the autoionization threshold. The inset illustrates the spread of energies of the electrons used in these experiments. 
Much astrophysical information comes from the study of spectral lines which arise from the excitation of positive ions by electrons and it is usually the near-threshold region of the excitation function which contributes most to the population of excited states.

\section{Detachment of Electrons from $\mathrm{H}^{-}$by Electron or Proton Impact}

The opacity of photospheres to visible radiation depends largely upon the concentration of $\mathrm{H}^{-}$ions which is given by Saha's equation only if local thermodynamic equilibrium prevails. To determine whether this assumption is valid, cross-sections are needed for the processes which lead to the formation and destruction of $\mathrm{H}^{-}$(e.g. Branscomb, 1967). Following a chequered series of calculations, the cross-sections for,

$$
\mathrm{H}^{-}+e \rightarrow \mathrm{H}+2 e
$$

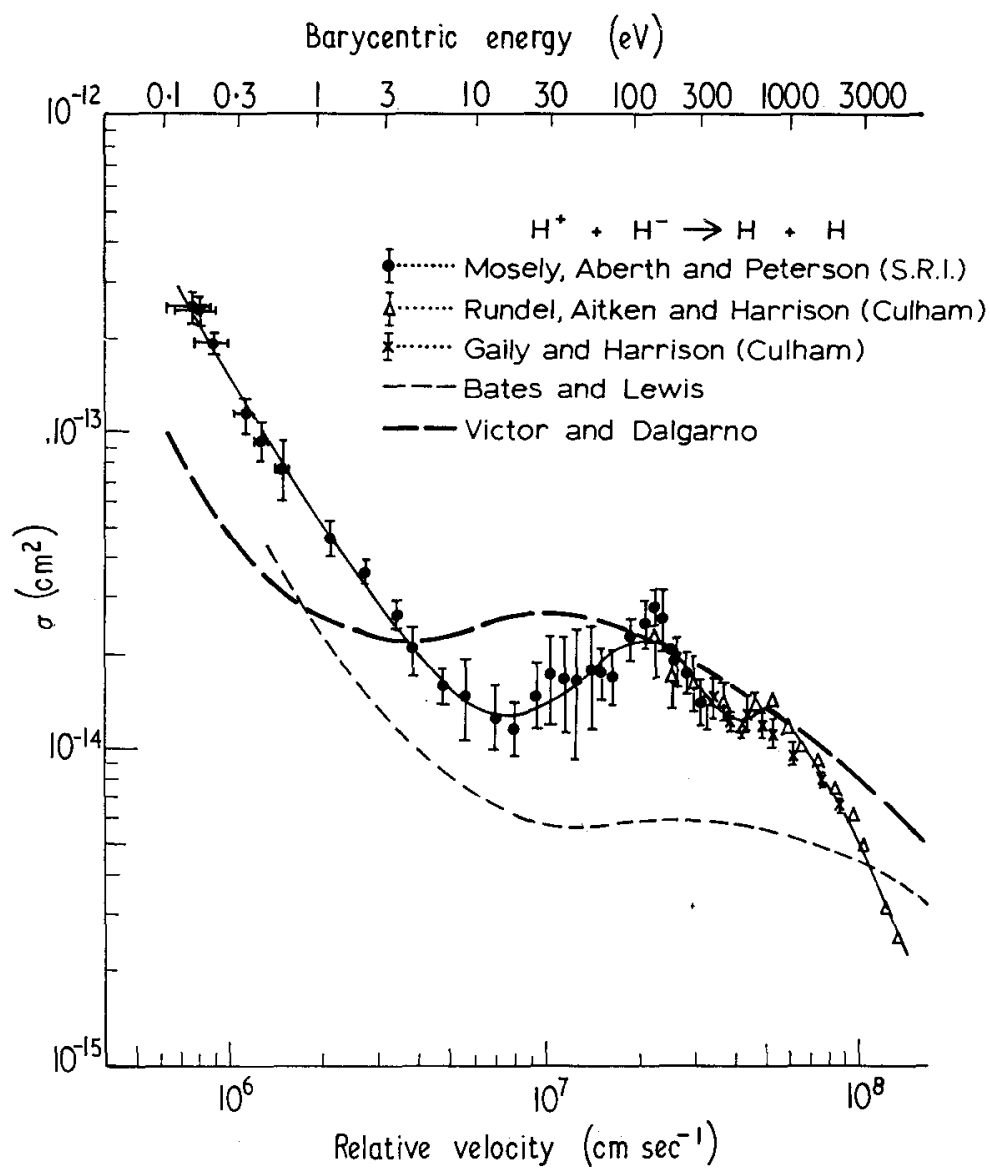

Fig. 8. Measurements and calculations of charge-changing collisions between $\mathrm{H}^{+}$and $\mathrm{H}^{-}$ions. The broken curves illustrate theoretical results by Bates and Lewis and by Victor and Dalgarno. 
was measured by Tisone and Branscomb (1966, 1968), and Dance et al. (1967). A new discussion was, however, provoked (e.g. Branscomb, 1969) by disparities between these measurements at the higher energies and the fact that Tisone and Branscomb's results were inconsistent with calculations performed by Inokuti and Kim (1968) in the Bethe approximation. The situation has very recently been resolved by Peart et al. (to be published) who made new observations which, at high electron energies, agree closely with Dance et al. and with theory. It seems that Tisone and Branscomb considerably overestimated the cross-section but only at energies greater than about $200 \mathrm{eV}$.

Recent measurements by Harrison and his colleagues $(1969,1970)$ and by Moseley et al. (1970) of cross sections for,

$$
\mathrm{H}^{+}+\mathrm{H}^{-} \rightarrow \mathrm{H}+\mathrm{H}
$$

are exceptionally interesting because they clearly demonstrate the inadequacy of the Landau-Zener approximation. Experimental and theoretical results are compared in Figure 8 .

Although the destruction of $\mathrm{H}^{-}$by electrons or protons is not sufficiently rapid for it to be of major astrophysical interest, the results might encourage a deeper understanding of negative ions. The most rapid destruction of $\mathrm{H}^{-}$in the solar photospheres appears to follow from associative detachment,

$$
\mathrm{H}+\mathrm{H}^{-} \rightarrow \mathrm{H}_{2}+e
$$

for which rate coefficients have been measured by Schmeltekopf et al. (1967).

\section{References}

Bacon, F. M. and Hooper, J. W.: 1969, Phys. Rev. 178, 182.

Bely, O.: 1968, J. Phys. B. (2), 1, 23.

Branscomb, L. M.: 1967, Proc. 5th Int. Conf. Phys. Elec. and Atomic Collins., Leningrad.

Branscomb, L. M.: 1969, Physics of One-and Two-Electron Atoms (Ed. by F. Bopp and H. Kleinpoppen), North-Holland Publ. Co., Amsterdam.

Dance, D. F., Harrison, M. F. A., and Smith A. C. H.: 1966, Proc. Roy. Soc. A290, 74.

Dance, D. F., Harrison, M. F. A., and Rundel, R. D.: 1967, Proc. Roy. Soc. A299, 5251

Dolder, K. T.: 1969, Chapter 5 of Case Studies in Atomic Collision Physics (Ed. by E. W. McDaniel and M. R. C. McDowell), North-Holland Publ. Co., Amsterdam.

Drawin, H. W.: 1961, Z. Phys. 164, 513.

Dunn, G. H.: 1969, Article on 'Colliding Beams' in Atomic Physics, Plenum Press.

Economides, D. G. and McDowell, M. R. C.: 1969, J. Phys. B. (2), 2, 1323.

Gaily, T. D. and Harrison, M. F. A.: 1970, J. Phys. B. (2), 3, L25.

Harrison, M. F. A.: 1968, Methods of Exptl. Physics 7B (Ed. by B. Bederson and W. L. Fite), Academic Press, New York.

Inokuti, M. and Kin, Y. K.: 1968, Phys. Rev. 173, 154.

Kim, Y. K. and Inokuti, M.: 1969, Proc. 6th Int. Conf. Physics Elec. and Atomic Collns. MIT Press, Boston.

McFarland, R. and Kinney, J. D.: 1964, Phys. Rev. 137, A1058.

Martin, S. O., Peart, B., and Dolder, K. T.: 1968, J. Phys. B. (2), 1, 537.

Moores, D. L. and Nussbaumer, H.: 1970b, J. Phys. B. (2), 3, 168.

Moseley, J., Aberth, W., and Peterson, J. R.: 1970, Phys. Rev. Letters 24, 435.

Omidvar, K.: 1969, Phys. Rev. 177, 212. 
Peach, G.: 1968, J. Phys. B. (2) 1, 1088.

Peach, G.: 1970, J. Phys. B. (2), 3, 328.

Peart, B. and Dolder, K. T.: 1968a, J. Phys. B. (2), 1, 240.

Peart, B. and Dolder, K. T.: 1968b, J. Phys. B. (2), 1, 872.

Peart, B., Martin, S. O., and Dolder, K. T.: 1969a, J. Phys. B. (2), 2, 1176.

Peart, B., Walton, D. S., and Dolder, K. T.: 1969b, J. Phys. B. (2), 2, 1347.

Peart, B., Walton, D. S., and Dolder, K. T.: J. Phys. B. (to be published).

Rundel, R. D., Aitken, K. L., and Harrison, M. F. A.: 1969, J. Phys. B. (2), $2,954$.

Schmeltekopf, A. L., Fehsenfeld, F. C., and Ferguson, E. E.: 1967, Astrophys. J. 148, L155.

Schram, B. L., Boerboom, A. J. H., and Kistenmaker, J.: 1966a, Physica 32, 185.

Schram, B. L., Moustafa, H. R., Schutten, J., and de Heer, F. J.: 1966b, Physica 32, 734.

Tisone, G. C. and Branscomb, L. M.: 1966, Phys. Rev. Letters 17, 236.

Tisone, G. C. and Branscomb, L. M.: 1968, Phys. Rev. 170, 169. 